\title{
Cytotoxic Effect of Hypoxic Environment in Mesenchymal Stem Cell ${ }^{\dagger}$
}

\author{
Sevil Özer ${ }^{1}$, H. Seda Vatansever ${ }^{2,3}$ and Feyzan Özdal-Kurt 1,4,* \\ 1 Faculty of Sciences and Letters, Department of Biology, Manisa Celal Bayar University, Manisa 45140, \\ Turkey; sevilguner89@gmail.com \\ 2 Faculty of Medicine, Department of Histology and Embryology, Manisa Celal Bayar University, \\ Manisa 45020, Turkey; sedavatansever@yahoo.com \\ 3 Experimental Health Research Center of Health Sciences, Near East University, Nicosia 45020, Cyprus \\ 4 Department of Molecular, Cell and Systems Biology, Stem Cell Center, College of Natural and Agricultural \\ Sciences, University of California Riverside, Riverside 92512, CA, USA \\ * Correspondence: feyzanozdalkurt@yahoo.com or feyzank@ucr.edu; Tel: +1-951-961-0405 \\ + Presented at the 2nd International Cell Death Research Congress, Izmir, Turkey, 1-4 November 2018.
}

Published: 12 December 2018

\begin{abstract}
Bone marrow mesenchymal stem cells (BM-MSCs) are used to repair hypoxic or ischemic tissue. After hypoxic the level of ATP is decreases, cellular functions do not continue and apoptosis or necrosis occur. Apoptosis is a progress of programmed cell death that occurs in normal or pathological conditions. In this study, we were investigated the hypoxic effect on apoptosis in mesenchymal stem cell. Bone marrow-derived stem cells were cultured in hypoxic $(1 \%$ or $3 \%)$ or normoxic conditions 24, 96 well plates for $36 \mathrm{~h}$. Cell viability was shown by MTT assay on $36 \mathrm{~h}$. After fixation of cells with $4 \%$ paraformaldehyde, distributions of caspase-3, Bcl-2 and Bax with indirect immunoperoxidase technique, apoptotic cells with TUNEL assay were investigated. All staining results were evaluated using H-score analyses method with ANOVA, statistically. As a result, hypoxic condition was toxic for human mesenchymal stem cells and the number of death cell was higher in that than normoxic condition.
\end{abstract}

Keywords: stem cells; hypoxic; cytotoxicity

\section{Introduction}

Mesenchymal stem cells derived from bone marrow (BM-MSCs) are adult multipotent stem cells with the self-renewing capacity and the ability to differentiate into cells of various connective tissue lineages. They are regarded as a promising and potential alternative source in the repair of many cells and tissues due to its multilineage differentiation capability into not only mesoderm but also ectodermic and endodermic lineages such as osteoblasts, chondrocytes, adipocytes, neurocytes and myoblasts $[1,2]$. In addition, MSCs are play a role to controlling of other cells such as cancer cells when it is in their microenvironment to survival or death. Therefore, the condition for MSCs really critical for their function and secreting molecules which effecting other cell types. The low oxygen concentration in the stem cell niche or physiological microenvironment is an important component in the maintenance of MSC properties such as self-renewal, multipotency, and extended survival $[3,4]$. Stem cells are more resistant to the hypoxic environment than somatic cells. The reason for this is that they have differentiation capacities and have the ability to renew themselves $[5,6]$. Therefore, the analyses of MSCs properties in hypoxic condition may to be help to understand their functions in different microenvironment. In this study we aimed to analyze of BM-MSCs survival potential in hypoxic condition. 


\section{Materials and Methods}

Hypoxic and normoxic cell culture of human BM-MSCs were performed. For hypoxic condition, hypoxic chamber (Stem Cell Tech., 27310, Vancouver, BC, Canada) was used according to manufacturer's instructions. Gas mixtures were prepared to $1 \%$ or $3 \% \mathrm{O}_{2}$ levels. As a control group, $5 \% \mathrm{CO}_{2}$ humidified environment was used to provide normoxic conditions $\left(20 \% \mathrm{O}_{2}\right)$ for $36 \mathrm{~h}$ in an incubator (ESCO, CCL-170B-8, Singapore).

hBM-MSCs cells was purchased from the CLS (CLS Cell Lines Service GmbH, Eppelheim, Germany). hBM-MSCs were cultured in Minimum Essential Medium Eagle-alpha modification $(\alpha-$ MEM, CP15-1157, Capricorn Scientific, Ebsdorfergrund, Germany) supplemented with $10 \%$ fetal bovine serum (FBS, 10500-64, Gibco, Grand Island, NY, USA), 1\% penicillin-streptomycine (15140122, Gibco, NY, USA). After $36 \mathrm{~h}$ incubation of cells, the cell viability was detected by MTT, 3-(4,5dimethylthiazol-2-yl)-2,5-diphenyltetrazolium bromide (M5655, Millipore Sigma, St. Louis, MO, USA). The absorbance was measured at $570 \mathrm{~nm}$ using an UV-vis spectrophotometer multi-plate reader (UNICAM, Uv visible Spectrofotometre, Leighton Buzzard, UK). After $36 \mathrm{~h}$ incubation, the cells were fixed with $4 \%$ paraformaldehyde (818715, Millipore Sigma, Burlington, MA, USA) and distribution of anti-Caspase 3 (ab2302, Abcam, Cambridge, MA, USA), anti-Bcl-2 (DB001, Delta Biolab, Gilroy, CA, USA), anti Bax (sc-7480, Santa Cruz, Dallas, TX, USA) and Cytochrome-c (sc13156, Santa Cruz, Dallas, USA) were analyzed with indirect immunoperoxidase staining. DNA fragmentation was detected by terminal deoxynucleotidyl transferase-biotin nick end-labelling (TUNEL) method with a commercial in situ apoptosis detection kit (Apoptag Plus Peroxidase In Situ Apoptosis Detection Kit, S7101, Millipore Sigma, Massachusetts, USA), according to the manufacturer's instructions. Results evaluated with the One-Way ANOVA statistical test.

\section{Results}

hBM-MSCs were cultured under hypoxic $5 \% \mathrm{CO}_{2}, 3 \% \mathrm{O}_{2}$ and $94 \% \mathrm{~N}_{2}$ or normoxic condition for $36 \mathrm{~h}$. The proliferation rate of hBM-MSCs cultured under 3\% hypoxic was higher than that of hBMMSCs cultured under 1\% hypoxic condition but less than the normoxic condition (Figure 1).

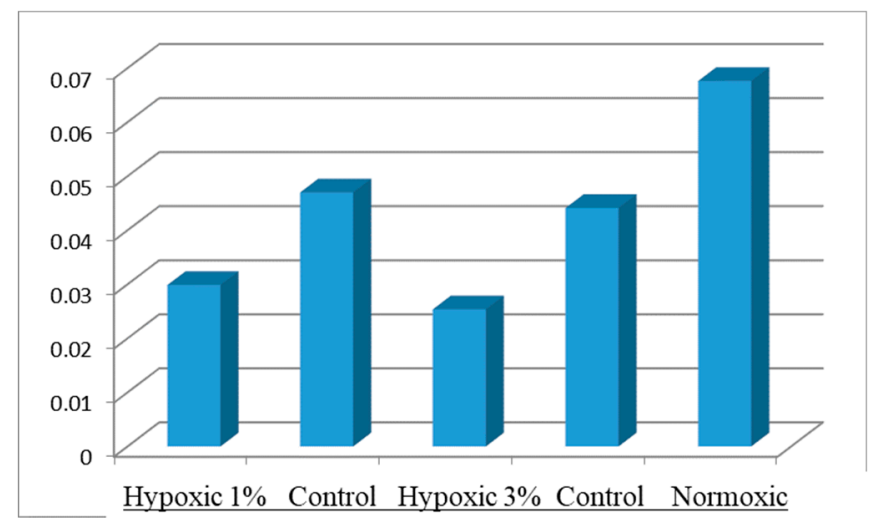

Figure 1. Cell viability of hBM-MSCs under hypoxic and normoxic conditions at $36 \mathrm{~h}$ of culture.

According to immunocytochemical analyses, hBM-MSCs under hypoxic conditions were showed strong positive staining of Caspase-3 immunoreactivity while it was negative in control group (Figure 2). Bcl-2 immunoreactivity was negative in all hypoxic group (Figure 2). Bax immunoreactivity was also moderate to strong in all hypoxic group (Figure 2). 
$1 \%$ Hypoxic $\quad 3 \%$ Hypoxic Normoxic
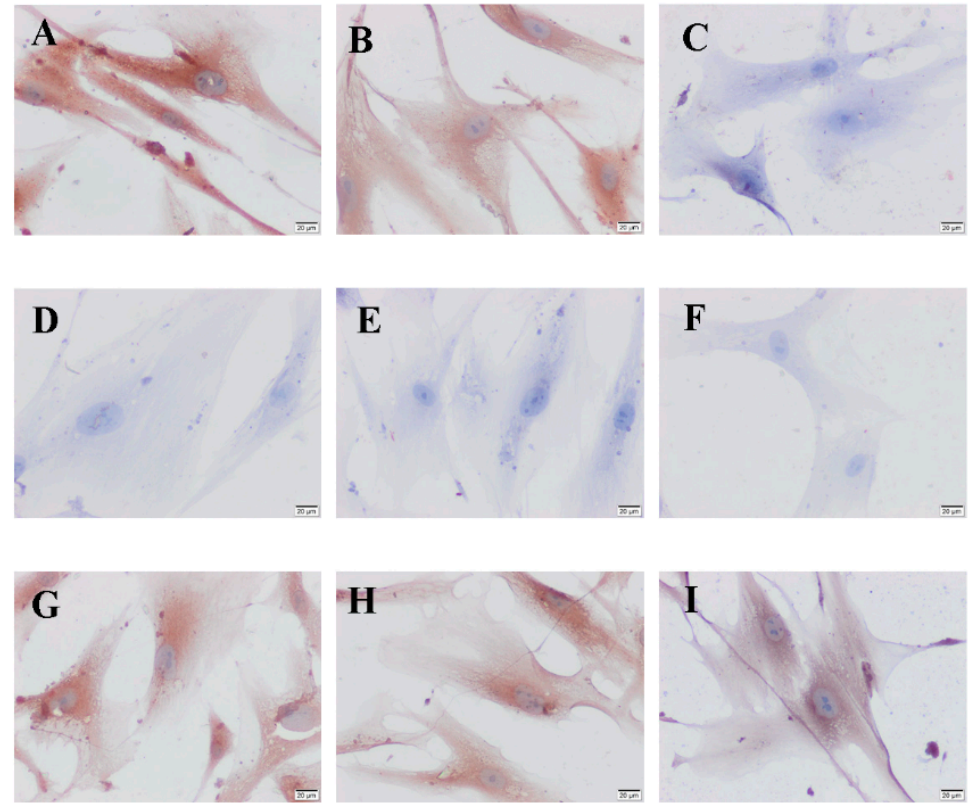

Figure 2. The distribution of caspase-3 (A-C), Bcl-2 (D-F) and Bax (G-I) immunreactivities of 1\%, 3\% hypoxic and normoxic groups of hBM-MSCs. Scale bars: $20 \mu \mathrm{m}$.

When compare of the H-Score analysis of caspase-3, Bcl-2 and Bax immunoreactivity was found to be similar with $3 \%$ hypoxic group of staining of caspase- 3 in $1 \%$ hypoxic group and it was not statistically significant $(p>0.005)$. It was found that the increase in caspase-3 immunoreactivity was higher in both $1 \%$ hypoxic and 3\% hypoxic groups than in normoxic group, and it was found statistically significant $(p<0.0001)$. Since Bcl-2 immunoreactivity was negative in all groups, it could not be compared statistically. Bax immunoreactivity was positive in all three groups, after distribution of both hypoxic $1 \%$ and $3 \%$ hypoxic groups were similar all groups and it was not statistically significant $(p>0.005)$ (Table 1).

Table 1. H-Score analysis of Caspase-3, Bcl-2 and Bax immunocytochemical distributions in hypoxic $(1 \%$ and $3 \%)$ and normoxic groups. Values (mean \pm SD) were given, $p<0.05$ was considered as significant after the comparison of the values and was shown with $\left(^{*}\right)$.

\begin{tabular}{cccc}
\hline HSCORE & Caspase-3 & Bcl-2 & Bax \\
\hline Hypoxic 1\% & $388.3 \pm 11^{*}$ & 0 & $343 \pm 7.394$ \\
\hline Hypoxic 3\% & $389.5 \pm 21^{*}$ & 0 & $348 \pm 35.64$ \\
\hline Normoxic & 0 & 0 & $224.8 \pm 17.91^{*}$ \\
\hline
\end{tabular}

The TUNEL-positive cells were observed in all groups (Figure 3).

$$
1 \% \text { Hypoxic } \quad 3 \% \text { Hypoxic } \quad \text { Normoxic }
$$

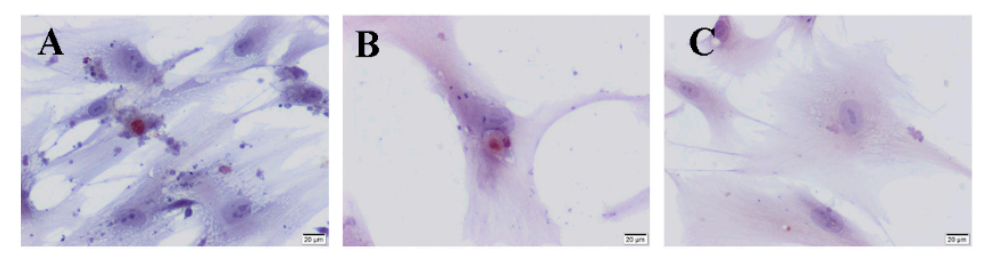

Figure 3. TUNEL staining of hBM-MSCs under hypoxic (A,B) and normoxic (C) conditions. Scale bars: $20 \mu \mathrm{m}$. 
After compared of between all groups, the number of TUNEL-positive cells was higher in $\% 1$ hypoxic and this increase was statistically significant when compared with normoxic group $(p=$ $0.0027)$. However, in $\% 3$ hypoxic, the number of TUNEL-positive cell was not statistically significant $(p=0.4115)$. It was found that the fewest TUNEL-positive cell number was in normoxic group and it was statistically significant after comparison with in $1 \%$ and $3 \%$ hypoxic groups $(p=0.0027, p=0.0369$, respectively) (Figure 4).

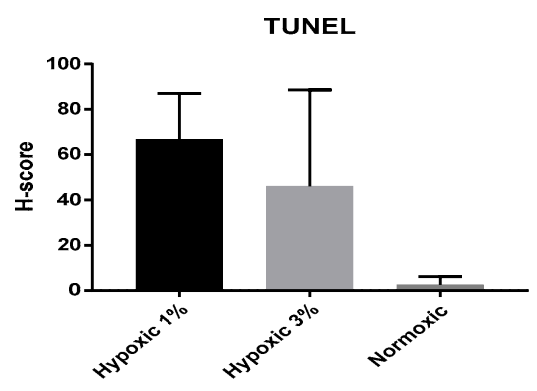

Figure 4. Comparative data of TUNEL-positive cell number in hypoxic (1\% and 3\%) and normoxic groups.

\section{Discussion}

BM-MSCs are usually found as bone lining cells, they are exposed to considerably lower oxygen concentrations in vivo than in vitro. This situation has prompted several groups to investigate key features of MSCs with conflicting results [4]. Thus, oxygen tension seems to be an important regulatory factor in the proliferation, differentiation and matrix production of MSCs [5]. In our study, mitochondrial pathway mediated activated mesenchymal stem cell apoptosis was found to be increased with Bax and caspase-3. In conclusion, MSCs were affected in hypoxic environment and their deaths were triggered by apoptotic mechanisms.

Acknowledgments: This study was supported from Manisa Celal Bayar University BAP (Project number: 2017033).

\section{References}

1. Han, Y.S.; Lee, J.H.; Yoon, Y.M.; Yun, C.W.; Noh, H.; Lee, S.H. Hypoxia-induced expression of cellular prion protein improves the therapeutic potential of mesenchymal stem cells. Cell Death Dis. 2016, 7, e2395.

2. Taran, R.; Mamidi, M.K.; Singh, G.; Dutta, S.; Parhar, I.S.; John, J.P.; Bhonde, R.; Pal, R.; Das, A.K. İn vitro and in vivo neurogenic potential of mesenchymal stem cells isolated from different sources. J. Biosci. 2014, 39, 157-169.

3. Kim, D.S.; Ko, Y.J.; Lee, M.W.; Park, H.J.; Park, Y.J.; Kim, D.I.; Sung, K.W.; Koo, H.H.; Yoo, K.H. Effect of low oxygen tension on the biological characteristics of human bone marrow mesenchymal stem cells. Cell Stress Chaperones 2016, 21, 1089-1099.

4. Holzwarth, C.; Vaegler, M.; Gieseke, F.; Pfister, S.M.; Handgretinger, R.; Kerst, G.; Muller, I. Low physiologic oxygen tensions reduce proliferation and differentiation of human multipotent mesenchymal stromal cells. BMC Cell Biol. 2010, 11, 11.

5. Bae, H.C.; Park, H.J.; Wang, S.Y.; Yang, H.R.; Lee, M.C.; Han, H.S. Hypoxic condition enhances chondrogenesis in synovium-derived mesenchymal stem cells. Biomater. Res. 2018, 22, 28.

6. Huang, X.; Ding, L.; Bennewith, K.L.; Tong, R.T.; Welford, S.M.; Ang, K.K.; Story, M.; Le, Q.T.; Giaccia, A.J. Hypoxia-inducible mir-210 regulates normoxic gene expression involved in tumor initiation. Mol. Cell 2009, 35, 856-867.

(C) 2018 by the authors. Licensee MDPI, Basel, Switzerland. This article is an open access article distributed under the terms and conditions of the Creative Commons Attribution (CC BY) license (http://creativecommons.org/licenses/by/4.0/). 